\title{
Macular Pigment is Associated with Glare-Affected Visual Function and Central Visual Field Loss in Glaucoma
}

James Loughman

Technological University Dublin, james.loughman@tudublin.ie

We Fong Siah

Mater University Hospital, Dublin.

Colm O'Brien

University College Dublin

Follow this and additional works at: https://arrow.tudublin.ie/otpomart

Part of the Eye Diseases Commons

\section{Recommended Citation}

Siah WF., O'Brien C. \& Loughman J. (2017) Macular pigment is associated with glare-affected visual function and central visual field loss in glaucoma. $\mathrm{Br} \mathrm{J}$ Ophthalmol, Published Online First: [Oct 5, 2017].doi:10.1136/bjophthalmol-2017-310215

This Article is brought to you for free and open access by ARROW@TU Dublin. It has been accepted for inclusion in Articles by an authorized administrator of ARROW@TU Dublin. For more information, please contact arrow.admin@tudublin.ie, aisling.coyne@tudublin.ie, gerard.connolly@tudublin.ie.

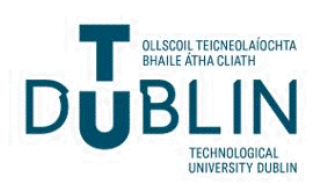




\title{
Macular pigment is associated with glare-affected visual function and central visual field loss in glaucoma
}

\author{
We Fong Siah, ${ }^{1}$ Colm O'Brien, ${ }^{1,2}$ James J Loughman ${ }^{3,4}$
}

\begin{abstract}
${ }^{1}$ Department of Ophthalmology, Mater Misericordiae University Hospital, Dublin, Ireland ${ }^{2}$ School of Medicine, University College Dublin, Dublin, Ireland ${ }^{3}$ School of Physics, Clinical and Optometric Sciences, College of Sciences and Health, Dublin Institute of Technology, Dublin, Ireland

${ }^{4}$ Faculty of Health Sciences, African Vision Research Institute, University of KwaZulu-Natal, Durban, South Africa
\end{abstract}

\section{Correspondence to}

We Fong Siah, Mater

Misericordiae University Hospital, Dublin 7, D07 R2WY, Ireland; wefong_siah@yahoo. com

Received 17 January 2017 Revised 3 September 2017 Accepted 21 September 2017

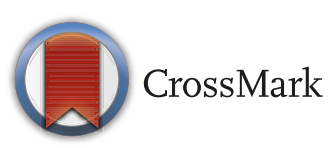

To cite: Siah WF, O'Brien $C$, Loughman JJ. Br J Ophthalmol Published Online First: [please include Day Month Year]. doi:10.1136/

bjophthalmol-2017-310215

\section{ABSTRACT}

Aim To evaluate the relationship between macular pigment optical density (MPOD) and glare disability in open-angle glaucoma.

Methods A cross-sectional analysis of baseline data ( 88 subjects; median age, 67 (range 36-84) years) collected during the Macular Pigment and Glaucoma Trial (ISRCTN registry number: 56985060$)$. MPOD at $0.25^{\circ}, 0.5^{\circ}$ and $1^{\circ}$ of retinal eccentricity was measured using customised heterochromatic flicker photometry. Mesopic contrast sensitivity with glare $(\mathrm{mCSg})$, photostress recovery time (PRT) and self-reported glare symptoms were evaluated. Fourier-domain optical coherence tomography was used to analyse ganglion cell complex (GCC) and identify foveal involvement.

Results Low spatial frequency $(f) \mathrm{mCSg}$ was significantly correlated with MPOD at $0.25^{\circ}$ (three cycles per degree (cpd): $r=0.25, p=0.04$ ) and $0.5^{\circ}$ (three cpd: $r=0.23, p=0.04$ ) of retinal eccentricity. Those with foveal GCC loss exhibited lower MPOD, had worse low spatial fmCSg ( $1.5 \mathrm{cpd}$ and $3 \mathrm{cpd}, \mathrm{p}=0.02$ each) and prolonged PRT $(p=0.02)$ in comparison with those without foveal involvement. The depth of central $10^{\circ}$ field loss was related to MPOD at all eccentricities ( $p<0.01$ for all). Those who reported glare symptoms had a significantly lower MPOD at all retinal eccentricities $\left(0.25^{\circ}\right.$ and $1^{\circ}$ : $p=0.05$ each; $0.5^{\circ}: p=0.04$ ), including those with foveal involvement $\left(0.25^{\circ}: p=0.05 ; 0.5^{\circ}: p<0.01 ; 1^{\circ}: p=0.01\right)$. Conclusions Macular pigment level may be an important consideration among those experiencing disability glare in glaucoma, including those with foveal involvement.

\section{INTRODUCTION}

Disability glare impairs vision when light scatters within the eye and casts a veiling luminance on the retina. This causes a loss of image contrast and decreases the visibility of objects near the source of glare. ${ }^{1}$ Individuals with glaucoma commonly suffer from disability glare, and this has been shown to be present even in those with mild visual loss. ${ }^{23}$ The aetiology of glare disability in glaucoma is poorly understood and, currently, little can be offered to the patient to alleviate this debilitating problem. Recent evidence pertaining to the involvement of the macula in glaucoma, ${ }^{4}$ however, has provided a rationale for the exploration of macular pigment (MP) in relation to functional (including glare related) visual loss associated with glaucoma.

MP is composed of the hydroxycarotenoids lutein, zeaxanthin and meso-zeaxanthin and is found to be highly concentrated at the central macula. ${ }^{5}$ Apart from its antioxidant properties and role as a filter for short wavelength blue light, it is also believed to play a beneficial role in visual performance. ${ }^{6-13}$ It has been shown that healthy individuals with higher macular pigment optical density (MPOD) experience less disability glare and demonstrate better photostress recovery times (PRT). ${ }^{78} 10$ Furthermore, oral dietary MP supplementation has been shown to increase MPOD and thereby improve glare disability and PRT in healthy individuals. ${ }^{9}$ Similar to glaucoma, individuals with age-related macular degeneration (AMD) commonly suffer from disability glare even in the presence of early disease. ${ }^{14}$ Dietary MP supplementation has also been shown to increase MPOD and thereby elicit an improvement in visual function including visual acuity (VA), contrast sensitivity (CS) and subjective glare recovery in AMD subjects. ${ }^{13}$

It has recently been demonstrated that MPOD is lower in glaucomatous eyes relative to age-matched normal eyes. ${ }^{15} 16$ Additionally, MPOD appears to be lowest among those glaucoma subjects with more severe structural damage and, in particular, in the presence of retinal ganglion cell complex loss involving the foveal region. ${ }^{17}$ Based on the evidence that lower MPOD may be associated with disability glare, this study was designed to evaluate whether MP may relate to functional performance in the glaucomatous eye.

\section{METHODS}

Subjects

This paper analyses the baseline glaucoma-related functional data that were collected from the Macular Pigment and Glaucoma Trial (ISRCTN registry number: 56985060). Information on this placebo-controlled, double-masked randomised trial is available online at http://www.isrctn.com. A total of 88 subjects with a diagnosis of openangle glaucoma were recruited. Written informed consent was obtained from all subjects, and the study adhered to the tenets of the Declaration of Helsinki.

All subjects with a diagnosis of open-angle glaucoma displayed glaucomatous optic disc cupping with compatible visual field (VF) loss and an open anterior chamber angle on gonioscopy. Four different types of open-angle glaucoma were considered for this trial: (1) primary open-angle glaucoma, where there is no associated ocular or systemic disease, (2) normal-tension glaucoma, where intraocular 
pressure is within the normal range, (3) pseudoexfoliative glaucoma, which is characterised by fibrillar protein deposition in the ocular anterior segment and (4) pigmentary glaucoma, which is characterised by excessive pigment deposition in the ocular anterior chamber angle.

The inclusion criteria were age $>18$ years, ETDRS LogMAR VA of 0.30 or better and subject ability to adhere to the trial protocol. Exclusion criteria comprised of any ocular disease such as AMD or moderate-to-significant cataract (Lens Opacity Classification System III (LOCS III) grades C3-5, P3-5, NC3-5 or NO3-5), previous ocular surgery other than cataract extraction or glaucoma drainage procedure, presence of a blue-filter intraocular lens, underlying diagnosis of diabetes mellitus, consumption of an oral dietary MP supplement in the past 6 months and the use of a miotic agent. The LOCS III $^{18}$ grades cataracts by type (cortical: C1-5 and posterior subcapsular: P1-5) and nuclear appearance (nuclear colour: NC1-6 and nuclear opalescence: NO1-6). We used the LOCS III standards were used to categorise eyes according to lens appearance as either 'mild cataract' (grades C1-2, P1-2, NC1-2 and/or NO1-2) or 'no cataract' (C0, P0, NC0 and/or NO0).

Details relating to age, gender, type of glaucoma, years diagnosed with glaucoma, history of smoking (current smoker, ex-smoker and never smoked), body mass index $\left(\mathrm{kg} / \mathrm{m}^{2}\right)$ and lens status (phakic vs pseudophakic) were recorded for each subject. All subjects also underwent a comprehensive slit lamp biomicroscopy examination. If both eyes met the inclusion criteria, the study eye was determined by random assignment using the software programme Research Randomizer (V.4.0). ${ }^{19}$

\section{Measurement of the MP spatial profile}

Heterochromatic flicker photometry (HFP) is a psychophysical technique for measuring MPOD that has been validated against the absorption spectrum of in vitro preparations of liposome-bound $\mathrm{MP}^{20}$ and shown to produce good test-retest reliability when compared with other methods of measuring MPOD. ${ }^{21}$ The HFP procedure is also minimally invasive, and pupil dilatation is not necessary. Furthermore, it has been shown that variation in pupil size has no effect on the final measurement and that the presence of cataract does not significantly affect the measurement. ${ }^{22}$ A customised HFP (cHFP) approach whereby the flicker frequency was optimised to take account of individual differences in vision as influenced by age, ocular disease or other factors was employed to optimise the task and thereby minimise the likely variance between readings. ${ }^{24}$ MPOD was measured at $0.25^{\circ}, 0.5^{\circ}$ and $1^{\circ}$ under conditions of dimmed light with a Macular Densitometer (Macular Metrics, Rehoboth, Massachusetts, USA) using the cHFP approach. Measurements taken at $7^{\circ}$ of eccentricity were used as a reference point where MPOD was assumed to be nil. Subjects were instructed to view a stimulus consisting of a square wave alternating blue $(460 \mathrm{~nm})$ and green $(550 \mathrm{~nm})$ flickering LED light source and were required to ascertain the point at which the flicker stopped or was minimally detectable (isoluminance match). For each test point, five readings were obtained to produce a mean MPOD at each eccentricity. Measurements were considered unreliable and excluded from data analysis if there was a large variance between measures $(\mathrm{SD}>0.05)$ for each test point.

\section{Dietary intake of lutein and zeaxanthin}

All glaucoma subjects completed the Lutein Zeaxanthin Questionnaire (Carotenoid \& Health Laboratory, Jean Mayer USDA Human Nutrition Center on Aging, Tufts University, Medford,
Massachusetts, USA), which has been used in other MP studies. ${ }^{1725}$ This semiquantitative food frequency questionnaire was used to quantify the dietary intake of lutein and zeaxanthin among glaucoma subjects in order to control for any disparity in MPOD between subgroups that may be caused by their respective diet.

\section{CS with glare}

The Optec 6500 device (Stereo Optical, Chicago, Illinois, USA) was used to objectively measure mesopic $\left(3.0 \mathrm{~cd} / \mathrm{m}^{2}\right)$ CS under glare conditions (mesopic contrast sensitivity with glare (mCSg)) in all subjects. All testing was carried out on natural size pupils under a constant ambient light. The Optec 6500 is based on the Functional Acuity Contrast Test, which consists of a series of sine-wave grating charts and is designed to test sensitivity across five spatial frequencies $(f)(1.5,3,6,12$ and 18 cycles per degree (cpd)) and nine levels of contrast in 0.15 log CS decrements. The in-built glare source consists of 12 white LEDs that are arranged circumferentially to the grating charts. The glare source was preset to provide a medium intensity luminance of 42 lux. ${ }^{1126}$ With distance glasses, if necessary, each subject was required to identify the orientation of the grating (right, up or left) without guessing, starting from the lowest spatial $f$ and the highest contrast. The last correct response for each spatial $f$ was plotted on a CS curve. When the subject was not able to see the highest contrast setting at any spatial $f$, the result was documented as half the lowest CS value for that spatial $f$.

\section{Photostress recovery}

PRT was measured using the portable, handheld MDD-2 Macular Adaptometer (Health Research Sciences, Lighthouse Point, Florida, USA) device, which has previously been used in other studies. ${ }^{27} 28$ It consists of a xenon flash source capable of producing a $200 \mu$ s duration flash that is subsequently filtered to block infrared and ultraviolet light before reaching the viewing eye. Each subject was instructed to hold the device up close to the study eye and to look through the $12 \mathrm{~mm}$ diameter viewing aperture, which contained an +8 dioptre-focusing lens. At the viewing aperture, the xenon flash reaches a peak irradiance of $4.5 \mathrm{~W} / \mathrm{cm}^{2}$, and the stimulus is of 0.41 -radian angular subtense. At the start of the test, it was ascertained that each subject was able to recognise a LED display number. The test was abandoned if it was not identifiable to the subject. Afterwards, a pushbutton was pressed to produce the xenon flash. The subject was required to call out a new numerical stimulus (single digit) that appeared simultaneous to the flash. This prompted the examiner to press a button to end the test. The time taken for the subject to correctly identify the stimulus was recorded as the PRT. If the response was incorrect, the test was repeated approximately 20 min later. An upper PRT limit of $60 \mathrm{~s}$ was incorporated into the device, which automatically resets for a new test thereafter. In the event that the subject did not respond within $60 \mathrm{~s}$, the test was abandoned.

\section{Glare symptoms}

In the evaluation of subjective glare symptoms, the subjects were asked the following question: 'Do you suffer from glare?'. Examples of different scenarios (glare from a poorly shielded street lamp in a dark street; glare from an oncoming car headlight while driving at night; and glare from low-lying sun in the winter) were used to provide context and assist them in answering this question better. 


\section{VF assessment}

The Swedish Interactive Threshold Algorithm Standard 24-2 and 10-2 programmes available in the Humphrey Visual Field Analyser (HVFA II, Carl Zeiss Meditec, Jena, Germany) were used to assess differential light sensitivity as a measure of global glaucomatous VF loss and residual visual function in the central retina (within $10^{\circ}$ of fixation), respectively. Mean deviation (MD) was used to define the severity of glaucoma (extent of functional loss) and was included in data analysis. Unreliable VF plots, as determined by a fixation loss of $>33 \%$ and/or false positive/negative rates $>20 \%$, were excluded from analysis.

$\mathrm{MP}$ is most concentrated within the central $1^{\circ}$ of the fovea. Although the 24-2 test pattern is useful in providing a global scale of glaucomatous VF loss, it uses a $6^{\circ} \times 6^{\circ}$ grid, which only has four points within the central $4.2^{\circ}$ radius of fixation. In contrast, the $10-2$ test pattern uses a $1^{\circ} \times 1^{\circ}$ grid, with the central four points corresponding to within $1.4^{\circ}$ of the foveal centre. The finding of a glaucomatous depression involving any of the central four points of the 10-2 pattern deviation plots was recorded (foveal visual loss). For the study of MP and central visual function, we will focus on the MD of the 10-2 test for the purpose of statistical analysis.

\section{Fourier-domain optical coherence tomography (FD-OCT)}

The ganglion cell complex (GCC) protocol from the RTVue FD-OCT system (software V.5.1; Optovue, Fremont, California, USA) was used to categorise subjects' glaucoma by foveal involvement. This method had been described in detail previously. ${ }^{17} \mathrm{In}$ the GCC significance map, GCC thickness is colour coded by the significance of GCC thickness reduction (red, $\mathrm{p}<1 \%$; yellow, $\mathrm{p}<5 \%$; green, $\mathrm{p} \geq 5 \%$ ). It was classified as 'fovea-not-involved' (ie, no GCC loss encroaching the fovea) if the perimeter of the fovea zone was green in colour, and as 'fovea-involved' (ie, GCC loss encroaching the fovea) if the red scale encroached up to the perimeter of the fovea zone. The GCC subgroups allowed us to compare visual function by foveal involvement, which was previously shown to be associated with significantly lower MPOD.

\section{Statistical analysis}

The SPSS statistical software package (V.22.0; IBM, New York, USA) was used for data analysis. All variables were tested for normality using either Kolmogorov-Smirnov test (if $\mathrm{n}>50$ ) or Shapiro-Wilk test (if $\mathrm{n} \leq 50$ ). All data are presented as mean \pm SD for normally distributed data and as median (range) for non-normal distributions. Parametric tests (Pearson correlation coefficient and independent t-test) or non-parametric tests (Spearman Rho test and Mann-Whitney U-test) were carried out where appropriate. As the presence of a cataract can influence $\mathrm{mCSg}$ results, we controlled for this factor in our analysis. Linear regression analysis was also performed; $\mathrm{R}^{2}$ value signifies the percentage of variability in the dependent variable that can be explained by the model with the independent variable (MPOD). When comparing categoric data, a $\chi^{2}$ test was used. A 5\% statistical significance level of was adopted throughout the analysis.

\section{RESULTS}

Successful MPOD measurement was obtained for 69 subjects $(78.4 \%)$ at $0.25^{\circ}$ retinal eccentricity, 81 subjects $(92 \%)$ at $0.5^{\circ}$ eccentricity and 59 subjects $(67 \%)$ at $1^{\circ}$ of eccentricity. No significant relationship was observed between MPOD and potential confounders for any relationship that might exist between MPOD, visual function and other demographic parameters, including gender, time diagnosed with glaucoma, type of

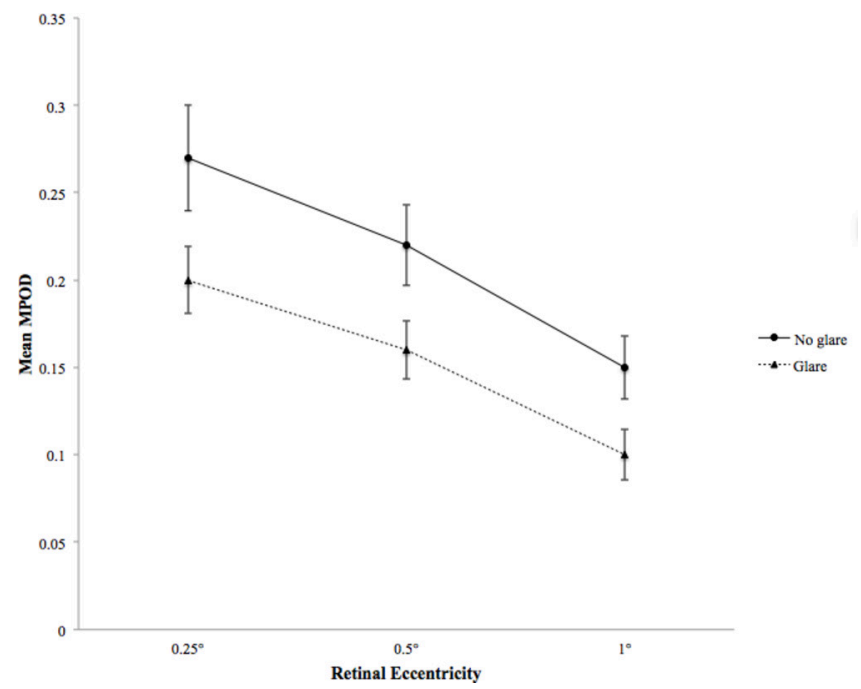

Figure 1 Graph demonstrates lower MPOD levels in those with symptoms of glare compared with those who were symptom free. MPOD, macular pigment optical density.

glaucoma, body mass index, lens status (mild cataract: yes or no) or smoking status ( $\mathrm{p}>0.05)$, except for age, which demonstrated a statistically significant relationship with MPOD at $0.5^{\circ}$ of retinal eccentricity only $(\mathrm{r}=-0.23, \mathrm{p}=0.04)$. Subsequent analyses involving MPOD at $0.5^{\circ}$ of retinal eccentricity were corrected for age.

\section{Glare and photostress recovery}

With controlling for the presence of mild cataract, MPOD was found to be statistically significantly correlated with $\mathrm{mCSg}$ for low spatial $\mathrm{fs}$ only, at both $0.25^{\circ}(3 \mathrm{cpd}$ : $\mathrm{r}=0.25, \mathrm{p}=0.04)$ and $0.5^{\circ}$ ( 3 cpd: $\left.r=0.23, p=0.04\right)$ of retinal eccentricity. No significant correlation was found between MPOD at $1^{\circ}$ and $\mathrm{mCSg}$. Linear regression analyses showed a low $\mathrm{R}^{2}$ value between MPOD and mCSg for low spatial fs (range 3\%-7\%). MannWhitney U-test was performed to evaluate any possible effect of cataract on glare, and no significant differences in mCSg were found at any $f$ between those with and those without mild cataract ( $p$ values range $0.75-1.0$ ).

In response to the glare symptoms question, 61\% $(n=54)$ of subjects stated that they suffer from glare symptoms, with a median duration of 4.5 years (range $0.5-30$ ). Those who suffered from glare symptoms had a significantly lower MPOD at all eccentricities relative to those without glare symptoms (figure 1), but no difference in terms of age, gender, length of glaucoma diagnosis, body mass index, lens status or dietary intake of lutein and zeaxanthin (table 1).

Valid PRT data were available for 73 subjects with a median of $19 \mathrm{~s}$ (range 8-59). The remaining subjects were either unable to see the display number in the Macular Adaptometer $(n=4)$ or to respond within the $60 \mathrm{~s}$ limit following the xenon flash $(n=11)$. There was no correlation between MPOD and PRT ( $p>0.05$ for all).

\section{VF loss}

HVF 24-2 MD was significantly correlated with MPOD $(r=0.33$, $\mathrm{p}<0.01$ at $0.25^{\circ} ; \mathrm{r}=0.33, \mathrm{p}<0.01$ at $0.5^{\circ} ; \mathrm{r}=0.31, \mathrm{p}=0.02$ at $1^{\circ}$ ). The median MD for HVF $10-2$ was $-7.85 \mathrm{~dB}$ with a range of -0.28 to -30.9 . Using the $10-2$ pattern deviation plots, we found $51.5 \%(n=45)$ of subjects without foveal visual loss in contrast to $48.9 \%(n=43)$ with foveal visual loss. However, 


\begin{tabular}{|c|c|c|c|}
\hline & No glare & Glare & $\mathrm{p}$ \\
\hline \multicolumn{4}{|l|}{ MPOD } \\
\hline $0.25^{\circ}$, mean \pm SD & $0.27 \pm 0.15$ & $0.20 \pm 0.13$ & $0.05^{*}$ \\
\hline $0.5^{\circ}$, mean $\pm S D$ & $0.22 \pm 0.13$ & $0.16 \pm 0.12$ & $0.04^{*}$ \\
\hline $1^{\circ}$, median (range) & $0.15(0-0.28)$ & $0.09(0-0.25)$ & $0.05+$ \\
\hline Age, mean $\pm S D$ & $64.5 \pm 9.6$ & $66 \pm 10.4$ & $0.51^{*}$ \\
\hline \multicolumn{4}{|l|}{ Gender } \\
\hline Male, n (\%) & $16(47.1)$ & $32(59.3)$ & \\
\hline Female, $\mathrm{n}(\%)$ & $18(52.9)$ & $22(40.7)$ & $0.28 \ddagger$ \\
\hline $\begin{array}{l}\text { Length of glaucoma diagnosis, } \\
\text { median (range) (year) }\end{array}$ & $6(0.5-15)$ & $6.5(0.5-32)$ & $0.60 \dagger$ \\
\hline $\begin{array}{l}\text { Body mass index, median (range) } \\
\left(\mathrm{kg} / \mathrm{m}^{2}\right)\end{array}$ & $26.3(18.7-39)$ & $25.3(18.5-42)$ & $0.80 \dagger$ \\
\hline \multicolumn{4}{|l|}{ Lens status } \\
\hline Phakic, n (\%) & $30(40)$ & $45(60)$ & \\
\hline Pseudophakic, n (\%) & $4(30.8)$ & $9(69.2)$ & $0.56 \ddagger$ \\
\hline \multicolumn{4}{|l|}{ Dietary intake, median (range) (mg/dL) } \\
\hline Lutein & $0.85(0-13.2)$ & $0.7(0-5.2)$ & $0.52 \dagger$ \\
\hline Zeaxanthin & $0.1(0-1.1)$ & $0.1(0-1.2)$ & $0.15 t$ \\
\hline HVF 10-2 MD, median (range) (dB) & $\begin{array}{l}-7.85(-0.28 \text { to } \\
-24.12)\end{array}$ & $\begin{array}{l}-7.75(-1.02 \text { to } \\
-30.94)\end{array}$ & $0.44 \dagger$ \\
\hline
\end{tabular}

*Mann-Whitney U-test.

tIndependent t-test.

$\neq \chi^{2}$ test.

HVF, Humphrey visual field; MD, mean deviation; MPOD, macular pigment optical density; $p$, significance (two tailed).

none of the latter displayed the findings of depression of all four central points on the 10-2 pattern deviation plots suggesting the possibility of reliable subject fixation of the Macular Densitometer stimulus targets to within $1.4^{\circ}$ of the foveal centre. MPOD at all retinal eccentricities were positively and statistically significantly correlated to the total amount of central visual field loss as measured using the HVF $10-2 \mathrm{MD}\left(\mathrm{r}=0.33, \mathrm{p}<0.01\right.$ at $0.25^{\circ}$; $\mathrm{r}=0.33, \mathrm{p}<0.01$ at $0.5^{\circ} ; \mathrm{r}=0.32, \mathrm{p}=0.01$ at $1^{\circ}$ ) (figure 2). Linear regression analyses between MPOD $\left(0.25^{\circ}, 0.5^{\circ}\right.$ and $1^{\circ}$ of retinal eccentricity, respectively) and HVF 10-2 MD showed a $\mathrm{R}^{2}$ ranging from $10 \%$ to $12 \%$.

\section{GCC subgroups}

No confounding differences were found between the GCC subgroups for gender, body mass index, length of time diagnosed with glaucoma, dietary intake or smoking habits except age ('fovea-not-involved' vs 'fovea-involved', $62.6 \pm 10.4$ years vs $67.9 \pm 9.0, p=0.02$ ), which was accounted for in subsequent analyses. A general linear analysis confirmed that age did not have a significant effect on parameters such as low spatial $f$ mCSg, PRT, HVF 10-2 MD and self-reported glare symptoms ( $>0.05$ for all).
Low spatial $f$ mCSg was significantly worse in the 'fovea-involved' subgroup compared with those without foveal involvement ( 1.5 cpd: $Z=-2.26, p=0.02 ; 3$ cpd: $Z=-2.36, p=0.02$ ) (table 2). Additionally, the 'fovea-involved' subgroup had a prolonged PRT in comparison with the 'fovea-not-involved' subgroup $(Z=-2.41, p=0.02)$. In the 'fovea-involved' subgroup, low spatial $f \mathrm{mCSg}$ was positively and statistically significantly correlated with MPOD at $0.25^{\circ}(1.5 \mathrm{cpd}$ : $\mathrm{r}=0.37, \mathrm{p}=0.02 ; 3$ cpd: $\mathrm{r}=0.43, \mathrm{p}=0.01)$ and $0.5^{\circ}(1.5 \mathrm{cpd}: \mathrm{r}=0.29, \mathrm{p}=0.05 ; 3$ cpd: $r=0.41, p=0.01)$ of retinal eccentricity, while no significant correlation was observed at $1^{\circ}$ and higher mCSg spatial $f$ s. Of those with significant correlations, $\mathrm{R}^{2}$ was found to be between $8 \%$ and $20 \%$. No significant correlation was observed between MPOD and mCSg in the 'fovea-not-involved' subgroup ( $p>0.05$ for all). Furthermore, MPOD was significantly lower at all retinal eccentricities in those with foveal involvement who reported glare symptoms compared with those without glare symptoms $\left(0.25^{\circ}: \mathrm{p}=0.05, \mathrm{t}=-1.99 ; 0.5^{\circ}: \mathrm{p}<0.01, \mathrm{t}=-2.92\right.$; $\left.1^{\circ}: \mathrm{p}=0.01, \mathrm{t}=-2.63\right)$, while no significant difference was observed in the 'fovea-not-involved' subgroup ( $p>0.05$ for all). No significant correlation was observed between MPOD and PRT within either GCC subgroup ( $>00.05)$. Residual visual function, as determined by HVF $10-2 \mathrm{MD}$, was significantly worse $(\mathrm{Z}=-4.42, \mathrm{p}<0.001)$ in the 'fovea-involved' subgroup (median, $-12.17 \mathrm{~dB}$ (range -0.28 to -26.78 )) in comparison with the 'fovea-not-involved' subgroup (median, -4.42 (range -1.02 to -20.62$)$ ).

\section{DISCUSSION}

This study demonstrates for the first time that a relationship exists between MPOD and disability glare in the glaucomatous eye. Low MP levels appear to be associated with poorer $\mathrm{mCSg}$ at low spatial $f$ and with increased symptoms of disability glare. More interestingly, it appears that this relationship between MPOD, $\mathrm{mCSg}$ and glare symptoms in individuals with glaucoma is mediated by foveal involvement.

Glare-affected visual performance under mesopic conditions linearly correlates with the optical density of MP among glaucoma subjects, particularly at lower spatial $f$. Our finding that higher MP is associated with improved glare-related visual performance is consistent with other reports, ${ }^{79} 10$ although some studies have failed to replicate such findings for mesopic CS. ${ }^{12} 28$ The inconsistency in the results among studies may be explained by the variation in population demographics, disease status and study methodology. Mesopic conditions were prioritised herein due to previous observations that impaired dark adaptation and disability glare can be found in glaucoma, ${ }^{29} 30$ coupled with evidence that glare-affected CS in the home environment is compromised due to poorer lighting. ${ }^{31}$ Although the study of MP in the glaucomatous eye is novel, other studies of MP in the non-glaucomatous eye have indicated that higher MPOD
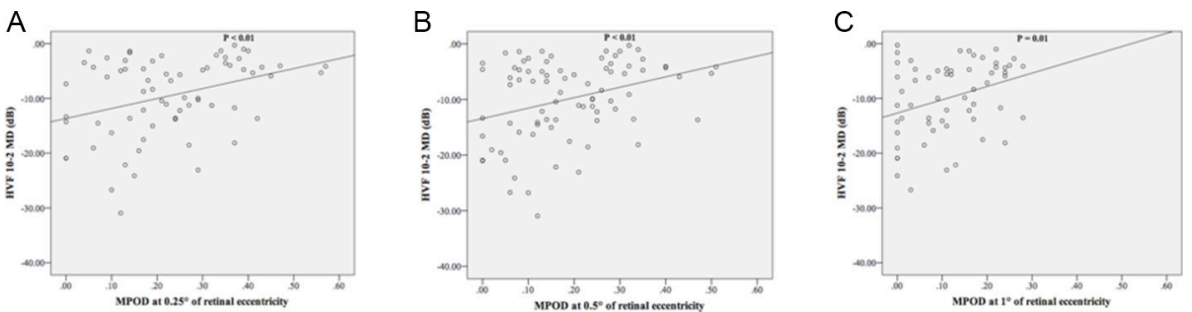

Figure 2 Scatter plots (A-C) show the correlation between MPOD and HVF 10-2 MD. HVF, Humphrey visual field; MD, mean deviation; MPOD, macular pigment optical density. 
Table 2 Comparison of visual function between GCC subgroups

\begin{tabular}{lccc}
\hline & Fovea-not-involved subgroup & Fovea-involved subgroup & $p$ \\
\hline mCSg, median (range) (cpd) & & $9(3.5-36)$ & 0.023 \\
\hline 1.5 & $13(3.5-50)$ & $10(5-40)$ & 0.018 \\
\hline 3 & $20(5-114)$ & $6(6-45)$ & 0.212 \\
\hline 6 & $6(6-85)$ & $4(4-8)$ & 0.127 \\
12 & $4(4-15)$ & 2 & 0.388 \\
\hline PRT, median (range) (s) & $2(2-4)$ & $20(9-58)$ & 0.015 \\
HVF 10-2 MD, median (range) (dB) & $15(8-59)$ & $-12.17(-0.28$ to -26.78$)$ & $<0.001$ \\
\hline
\end{tabular}

cpd, cycles per degree; GCC, ganglion cell complex; HVF, Humphrey visual field; mCSg, mesopic contrast sensitivity under glare condition; MD, mean deviation; PRT, photostress recovery time.

and oral dietary MP supplementation can be beneficial towards improving CS including CS under the influence of glare. ${ }^{7-10} 12$

Depressed levels of CS in the glaucomatous eye can be explained, in part, by the disturbance at the macula. ${ }^{32}$ Our finding that $\mathrm{mCSg}$ at low spatial $f$ significantly correlates with MPOD only in the 'fovea-involved' subgroup is not surprising as the fovea represents the most central anatomic location where MP density is highest. GCC thickness is linearly correlated with MPOD $^{16}{ }^{17}$; lower central MP in those with foveal-involved glaucoma may, therefore, be accountable, at least in part, for glare disability in these eyes. However, given the weak association between MPOD and mCSg at low spatial $f s$, there are other factors, in particular, retinal changes (retinal ganglion cells and photoreceptors) in the glaucomatous eye that require consideration. Studies have demonstrated an association between decreased CS in the glaucomatous eye and retinal ganglion cell dysfunction $^{33}$ or death. ${ }^{34}$ In addition, MP is localised to the photoreceptor and retinal ganglion cell layers. ${ }^{5}$ Therefore, the loss of retinal ganglion cells ${ }^{33} 34$ and photoreceptors, ${ }^{35}$ as encountered in glaucoma, can contribute to lower MPOD. We would like to highlight that the retinal factors affecting CS and storage of MP cannot be excluded in our experiments. Our finding of a relationship between MPOD and $\mathrm{mCSg}$ in the glaucomatous eye is novel and interesting, and future studies are required to interpret this further.

Those who reported the experience of symptomatic glare exhibited lower MPOD levels. This provides additional evidence that glare disability is potentially linked to residual MP levels in glaucoma. Our overall findings suggest that lower levels of MPOD in the glaucomatous eye might represent a contributory factor to the effects of reduced $\mathrm{mCSg}$ and symptomatic glare disability. Furthermore, we observed that the relationships between MPOD and both $\mathrm{mCSg}$ and glare symptoms respectively are isolated to those with foveal GCC loss. This suggests that further emphasis should be given to GCC analysis in glaucoma as foveal involvement relates to lower MPOD and poorer residual visual function. There is substantial evidence to support the role of MP in improving visual performance in healthy eyes $^{6-12}$ and in those with AMD. ${ }^{13}$ Oral dietary MP supplementation can augment MPOD and thereby effect an improvement in visual function such as disability glare, PRT and CS under glare conditions. ${ }^{91213}$ Further research is needed to investigate the therapeutic potential of oral dietary MP supplementation for improving glare disability especially in those with foveal-involved glaucoma.

There is strong evidence in the literature to suggest good structure-function relationship between macular RGC thinning and macular VF loss as measured by HVF $10-2 .{ }^{4}$ We included HVF $10-2$ as an ancillary test to capture macular function for this purpose. Our finding that HVF 10-2 MD was significantly worse in the foveal GCC loss subgroup in comparison with those without foveal involvement reinforces previous findings where MPOD positively correlated with GCC thickness and where those with foveal involvement displayed worse MPOD. ${ }^{16}{ }^{17}$ Although this study does not define the relationship between field loss, MPOD and glare-related visual performance, the correlation between central visual field loss and MPOD represents a novel finding in the field of glaucoma and worthy of further research.

The mechanisms underlying an abnormal PRT among individuals with glaucoma remains unclear. Our findings indicate that a relationship between MPOD and photostress recovery is confined to those with foveal-involved glaucoma, who demonstrated a prolonged PRT. In a study comparing healthy controls and those with diabetes mellitus (with and without diabetic retinopathy subgroups), there was no difference in PRT between groups. ${ }^{28}$ However, the study did not include those with diabetic maculopathy. There are conflicting results in the literature surrounding the relationship between MPOD and PRT. In a study of normal healthy controls, Stringham et $a l^{7}$ and Hammond et $a l^{10}$ each showed that MPOD correlated with photostress recovery in normal healthy controls while Loughman $e t a l^{11}$ reported otherwise. The variability of findings in the published literature may be explained by the differences in ocular pathology that were studied and the methods used to evaluate photostress recovery and MPOD, respectively.

There are limitations to this study. The HFP task can be challenging for some individuals, and this may affect the acquisition of MPOD measurements. We have applied stringent criteria and excluded readings that had large variances between them. This may have resulted in a high exclusion rate in the MPOD data that were available for statistical analysis. In the future, the use of fundus autofluorescence to measure MPOD will help eliminate this problem. ${ }^{23}$ Another concern regarding the use of HFP in measuring MPOD in the glaucomatous eye is the ability of the subject to fixate and report the absence of flicker in the stimulus targets. In this study, $48.9 \%$ of the subjects demonstrated some element of foveal visual field loss. This was determined by referring to the central four points (corresponding to $1.4^{\circ}$ of the foveal centre) of the 10-2 pattern deviation plots. As no subjects had evidence of light sensitivity depression in all central points, and as all subjects demonstrated acceptable fixation stability throughout the test, inability to reliably maintain central fixation of the stimulus targets was not likely to be an issue. Despite this, we do acknowledge that we cannot exclude the possibility that patients' glaucomatous foveal scotoma may have impacted their fixation capacity.

The lack of a healthy control group could be considered a drawback in this study. However, our main aim was to investigate 
the relationship between MPOD and glaucoma-related functional parameters, in particular glare disability, rather than the comparison of visual function between glaucoma and controls. The latter has previously been widely studied in the literature, and it is known that functional measures such as CS with and without glare and PRT are affected in those with glaucoma, and therefore was not the primary focus in this study. The cross-sectional nature of the study also limits the interpretation of the relationships that have been shown to exist but does serve to guide future research.

Those with 'fovea-involved' glaucoma were older in comparison with those without foveal involvement, although the age difference was small and not clinically meaningful. Coexisting ocular pathologies such as cataract and macular changes are more common with increasing age and may affect visual functions such as mCSg and PRT, respectively. We meticulously excluded individuals with moderate-to-significant cataract using the LOCS III grading, and therefore the possibility of a cataract as a confounding factor on $\mathrm{mCSg}$ was minimised. One possible limitation in this study is the absence of specific grading of the type and severity of mild cataract in this study. We showed, however, that cataract (those without cataract vs those with mild cataract) had no effect on mCSg in our study subjects. Furthermore, subsequent correlation analyses between MPOD and $\mathrm{mCSg}$ were also controlled for the presence of mild cataract, so this potential limitation is relatively minor. Any individuals with coexisting AMD were not recruited into this study. We also excluded those with an underlying diagnosis of diabetes mellitus as the finding of concurrent diabetic retinopathy has been linked with reduced MPOD. ${ }^{25}$ Any possible residual age-related confounding effects were controlled for in our statistical analysis, thereby negating the potential issue.

Another limitation of this study is the inability to disentangle the effects of disability and discomfort glare. Although the Optec 6500 device provides a consistent glare environment for our experiments, it is not discernible whether the measurements recorded are solely disability glare alone. Discomfort glare may be a contributing factor to participant experience of glare. Our finding that those with self-reported glare symptoms have lower MPOD relative to those without symptoms is a potentially important finding. Interpretation of this finding, however, needs to be tempered in relation to the nature of the question posed to participants. The question was qualitative in nature and not associated with a Likert or other scale to categorise responses. Furthermore, the response was not probed to elucidate additional detail as to the nature of the symptoms where present. Despite this limitation, our findings appear robust and therefore warrant consideration for future work.

\section{CONCLUSION}

This study extends previous findings that MPOD is lower in glaucoma ${ }^{15}$ and associated with structural ganglion cell complex losses at the fovea. ${ }^{17}$ It demonstrates that lower MPOD levels among glaucoma subjects is associated with (A) more severe glaucomatous VF loss, (B) poorer CS for low spatial $f$ stimuli under glare conditions and (C) self-reported symptoms of glare. More importantly, glaucoma-related psychophysical tests appear to be related to foveal GCC loss.

It is therefore possible to conclude that MP may have a role in the visual functional status of individuals with glaucoma, although a causal link has yet to be fully established. It is important to note that MP is only found in the central macula and therefore will not entirely explain the global symptom of disability glare but may be a contributing factor. The study of MP in glaucoma is of importance as the therapeutic potential to increase MPOD with an oral dietary MP supplement to improve disability glare is likely to appeal to patients with glaucoma exhibiting such symptoms should it work. To explore this concept further, the Macular Pigment and Glaucoma Trial has been designed to evaluate the MPOD response to an oral dietary MP supplement and any effects on glare in glaucoma.

Contributors Research design: all authors. Data acquisition and/or research execution: WFS. Data analysis and/or interpretation and manuscript preparation: all authors

Funding Howard Foundation.

Competing interests None declared.

Ethics approval Ethics approval was obtained from the local institutional review boards of the Mater Misericordiae University Hospital, Dublin and the Dublin Institute of Technology.

Provenance and peer review Not commissioned; externally peer reviewed.

(c) Article author(s) (or their employer(s) unless otherwise stated in the text of the article) 2017. All rights reserved. No commercial use is permitted unless otherwise expressly granted.

\section{REFERENCES}

1 Mainster MA, Turner PL. Glare's causes, consequences, and clinical challenges after a century of ophthalmic study. Am J Ophthalmol 2012;153:587-93.

2 Nelson P, Aspinall P, Papasouliotis 0, et al. Quality of life in glaucoma and its relationship with visual function. J Glaucoma 2003;12:139-50.

3 Goldberg I, Clement $\mathrm{Cl}$, Chiang TH, et al. Assessing quality of life in patients with glaucoma using the Glaucoma Quality of Life-15 (GQL-15) questionnaire. J Glaucoma 2009:18:6-12

4 Hood DC, Raza AS, de Moraes CG, et al. Glaucomatous damage of the macula. Prog Retin Eye Res 2013:32:1-21.

5 Trieschmann M, van Kuijk FJ, Alexander R, et al. Macular pigment in the human retina: histological evaluation of localization and distribution. Eye 2008;22:132-7.

6 Wooten BR, Hammond BR. Macular pigment: influences on visual acuity and visibility. Prog Retin Eye Res 2002;21:225-40.

7 Stringham JM, Garcia PV, Smith PA, et al. Macular pigment and visual performance in glare: benefits for photostress recovery, disability glare, and visual discomfort. Invest Ophthalmol Vis Sci 2011:52:7406-15.

8 Stringham JM, Hammond BR. The glare hypothesis of macular pigment function. Optom Vis Sci 2007:84:859-64.

9 Stringham JM, Hammond BR. Macular pigment and visual performance under glare conditions. Optom Vis Sci 2008;85:82-8.

10 Hammond BR, Fletcher LM, Elliott JG. Glare disability, photostress recovery, and chromatic contrast: relation to macular pigment and serum lutein and zeaxanthin. Invest Ophthalmol Vis Sci 2013;54:476-81.

11 Loughman J, Akkali MC, Beatty S, et al. The relationship between macular pigment and visual performance. Vision Res 2010:50:1249-56.

12 Loughman J, Nolan JM, Howard AN, et al. The impact of macular pigment augmentation on visual performance using different carotenoid formulations. Invest Ophthalmol Vis Sci 2012;53:7871-80

13 Liu R, Wang T, Zhang B, et al. Lutein and zeaxanthin supplementation and association with visual function in age-related macular degeneration. Invest Ophthalmol Vis Sci 2014; $56: 252-8$.

14 Scilley K, Jackson GR, Cideciyan AV, et al. Early age-related maculopathy and selfreported visual difficulty in daily life. Ophthalmology 2002;109:1235-42.

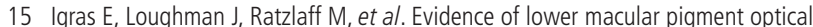
density in chronic open angle glaucoma. Br J Ophthalmol 2013;97:994-8.

16 Ji Y, Zuo C, Lin M, et al. Macular pigment optical density in Chinese primary open angle glaucoma using the one-wavelength reflectometry method. J Ophthalmol 2016:2016:2792103

17 Siah WF, Loughman J, O'Brien C. Lower macular pigment optical density in fovealinvolved glaucoma. Ophthalmology 2015;122.

18 Chylack LT, Wolfe JK, Singer DM, et al. The Lens Opacities Classification System III. The Longitudinal Study of Cataract Study Group. Arch Ophthalmol 1993;111:831-6.

19 Urbaniak GC, Plous S. 2013:Retrieve on. Research Randomizer (Version 4.0) [Computer software] from, 2013. http://www.randomizer.org/.

20 Bone RA, Landrum JT, Cains A. Optical density spectra of the macular pigment in vivo and in vitro. Vision Res 1992;32:105-10.

21 Hammond BR, Jr, Wooten BR, Snodderly DM. Individual variations in the spatial profile of human macular pigment. Journal of the Optical Society of America. A, Optics, image science, and vision 1997;14:1187-96.

22 Wooten BR, Hammond BR, Land RI, et al. A practical method for measuring macular pigment optical density. Invest Ophthalmol Vis Sci 1999;40:2481-9. 
23 Putnam CM. Clinical imaging of macular pigment optical density and spatial distribution. Clinical \& experimental optometry: journal of the Australian Optometrical Association 2017;100:333-40.

24 Stringham JM, Hammond BR, Nolan JM, et al. The utility of using customized heterochromatic flicker photometry (CHFP) to measure macular pigment in patients with age-related macular degeneration. Exp Eye Res 2008;87:445-53.

25 Scanlon G, Connell P, Ratzlaff M, et al. Macular pigment optical density is lower in type 2 diabetes, compared with type 1 diabetes and normal controls. Retina 2015;35:1808-16.

26 Nolan JM, Kenny R, O'Regan C, et al. Macular pigment optical density in an ageing Irish population: The Irish Longitudinal Study on Ageing. Ophthalmic Res 2010:44:131-9.

27 Newsome DA, Negreiro M. Reproducible measurement of macular light flash recovery time using a novel device can indicate the presence and worsening of macular diseases. Curr Eye Res 2009;34:162-70.

28 Loughman J, Ratzlaff M, Foerg B, et al. Suitability and repeatability of a photostress recovery test device, the macular degeneration detector (MDD-2), for diabetes and diabetic retinopathy assessment. Retina 2014;34:1006-13.
29 Velten IM, Horn FK, Korth M, et al. The b-wave of the dark adapted flash electroretinogram in patients with advanced asymmetrical glaucoma and normal subjects. Br J Ophthalmol 2001;85:403-9.

30 Glen FC, Crabb DP. Living with glaucoma: a qualitative study of functional implications and patients' coping behaviours. BMC Ophthalmol 2015;15:128.

31 Bhorade AM, Perlmutter MS, Wilson B, et al. Differences in vision between clinic and home and the effect of lighting in older adults with and without glaucoma. JAMA Ophthalmol 2013:131:1554-62.

32 Sun $\mathrm{H}$, Swanson WH, Arvidson B, et al. Assessment of contrast gain signature in inferred magnocellular and parvocellular pathways in patients with glaucoma. Vision Res 2008;48:2633-41.

33 Ventura LM, Porciatti V. Restoration of retinal ganglion cell function in early glaucoma after intraocular pressure reduction: a pilot study. Ophthalmology 2005;112:20-7.

34 Kerrigan-Baumrind LA, Quigley HA, Pease ME, et al. Number of ganglion cells in glaucoma eyes compared with threshold visual field tests in the same persons. Invest Ophthalmol Vis Sci 2000;41:741-8.

35 Nork TM, Ver Hoeve JN, Poulsen GL, et al. Swelling and loss of photoreceptors in chronic human and experimental glaucomas. Arch Ophthalmol 2000;118:235-45. 